\title{
EARLY RESULTS FROM THE GALACTIC O-STAR SPECTROSCOPIC SURVEY: C III EMISSION LINES IN Of SPECTRA
}

\author{
Nolan R. Walborn ${ }^{1}$, Alfredo Sota ${ }^{2}$, Jesús Maíz Apellániz ${ }^{2,6}$, Emilio J. Alfaro ${ }^{2}$, Nidia I. Morrell ${ }^{3}$, \\ Rodolfo H. Barbá ${ }^{4,7}$, Julia I. Arias ${ }^{4}$, ANd Roberto C. Gamen ${ }^{5}$ \\ ${ }^{1}$ Space Telescope Science Institute, 3700 San Martin Drive, Baltimore, MD 21218, USA; walborn@ stsci.edu \\ 2 Instituto de Astrofísica de Andalucía-CSIC, Glorieta de la Astronomía s/n, 18008 Granada. Spain; sota@iaa.es, jmaiz@iaa.es, emilio@iaa.es \\ ${ }^{3}$ Las Campanas Observatory, Observatories of the Carnegie Institution of Washington. Casilla 601. La Serena. Chile; nmorrell@1co.cl \\ ${ }^{4}$ Departamento de Física, Universidad de La Serena, Cisternas 1200 Norte, La Serena, Chile; rbarba@dfuls.cl, julia@dfuls.cl \\ ${ }^{5}$ Instituto de Astrofísica de La Plata-CONICET and Facultad de Ciencias Astronómicas y Geofísicas. Universidad Nacional de La Plata. Paseo del Bosque s/n, \\ 1900 La Plata, Argentina; rgamen@fcaglp.unlp.edu.ar \\ Received 2009 December 28: accepted 2010 February 5; published 2010 February 23
}

\begin{abstract}
On the basis of an extensive new spectroscopic survey of Galactic O stars, we introduce the Ofe category, which consists of normal spectra with $\mathrm{C}$ III $\lambda \lambda 4647-4650-4652$ emission lines of comparable intensity to those of the Of defining lines $\mathrm{N}$ III $\lambda \lambda 4634-4640-4642$. The former feature is strongly peaked to spectral type O5, at all luminosity classes, but preferentially in some associations or clusters and not others. The relationships of this phenomenon to the selective C III $\lambda 5696$ emission throughout the normal of domain, and to the peculiar, variable Of?p category, for which strong $C_{\text {III }} \lambda \lambda 4647-4650-4652$ emission is a defining characteristic, are discussed. Magnetic fields have recently been detected on two members of the latter category. We also present two new extreme Of?p stars, NGC 1624-2 and CPD $-28^{\circ} 2561$, bringing the number known in the Galaxy to five. Modeling of the behavior of these spectral features can be expected to better define the physical parameters of both normal and peculiar objects, as well as the atomic physics involved.
\end{abstract}

Key words: stars: early-type - stars: emission-line, Be - stars: fundamental parameters

\section{INTRODUCTION}

Comprising the most massive and energetic stars in the Galaxy, the Population I O spectral class is of considerable interest and has been the subject of many investigations. The optical spectra of over 1000 representatives have been observed. Their detailed spectral classification was begun by Plaskett \& Pearce (1931), who defined subtypes O5-O9 on the basis of helium-line ionization ratios, and denoted as Of the subset with selective emission lines of He II $\lambda 4686$ and $\mathrm{N}_{\text {III }} \lambda \lambda 4634-464($ ) 4642. This work was incorporated into the MK System (Johnson \& Morgan 1953; Abt et al. 1968; Morgan \& Keenan 1973), with the addition of luminosity classes V-I at subtypes O8-O9, and eventually of the earlier subtype O4. Further developments with higher photographic resolutions were introduced by Walborn (1971a, 1973a), Conti \& Alschuler (1971), and Conti \& Leep (1974), including luminosity classes at all subtypes, with the Of stars interpreted as the normal supergiants. The still earlier subtypes O3 and O2 were added by Walborn (1971b) and Walborn et al. (2002), respectively, and a digital atlas was provided by Walborn \& Fitzpatrick (1990). The complete historical and technical details of $\mathrm{OB}$ spectral classification have recently been reviewed by Walborn (2009).

Why then should further spectral classification work on the known Galactic O stars be undertaken? A major reason is the substantially superior quality and speed of modern CCD detectors, which render a complete, homogeneous, and higher signal-to-noise ratio $(\mathrm{S} / \mathrm{N})$ survey feasible. Such a study may be expected to improve the systematic and random accuracy of the classifications, while revealing new categories

\footnotetext{
6 Ramón y Cajal Fellow.

${ }^{7}$ Also at Instituto de Ciencias Astronómicas de la Tierra y del Espacio (ICATE-CONICET), Avenida España 1512 Sur. J5402DSP. San Juan. Argentina.
}

and peculiar objects within the sample. Moreover, further information about the endemic multiplicity of the $\mathrm{O}$ stars is essential for correct inference of their physical properties and evolution. Accordingly, we are conducting a massive digital survey of the optical spectra of all Galactic O stars in both hemispheres accessible to our available equipment as detailed below (Galactic O-Star Spectral Survey (GOSSS); J.M.A., overall/northern PI; R.H.B. and N.I.M., southern Co-PIs), i.e., to about 13th magnitude and 1300 stars. The first installment of the survey, including a new spectral classification atlas, will be presented by A. Sota et al. (2010, in preparation). Eventually, the data for all the stars will be linked to the public online Galactic O-Star Catalogue (GOSC; Maíz Apellániz et al. 2004). ${ }^{8}$ Here we present some initial examples of the unexpected developments that such a survey can produce.

\section{OBSERVATIONS}

The data have been acquired at three observatories and have similar albeit not identical characteristics. At the Observatorio de Sierra Nevada (OSN, Spain), the $1.5 \mathrm{~m}$ reflector and Albireo spectrograph with an $1800 \mathrm{l} \mathrm{mm}^{-1}$ grating provide a dispersion of $0.66 \mathrm{~A} \mathrm{pixel}^{-1}$. At the Centro Astronómico Hispano Alemán (CAHA, also Calar Alto Observatory, Spain), the $3.5 \mathrm{~m}$ reflector and TWIN spectrograph with a $1200 \mathrm{l} \mathrm{mm}^{-1}$ grating provide a dispersion of $0.54 \AA$ pixel $^{-1}$. At the Las Campanas Observatory (LCO, Chile), the $2.5 \mathrm{~m}$ Du Pont telescope and Boller \& Chivens spectrograph with a $1200 \mathrm{l} \mathrm{mm}^{-1}$ grating provide a dispersion of $0.80 \mathrm{~A} \mathrm{pixel}^{-1}$. The spectral resolving power is $\sim 3000$ in all three cases, and the typical S/N is 200-300. Further details about the data and reductions will be presented by A. Sota et al. (2010, in preparation). The stars and observations to be discussed here

\footnotetext{
8 http://dae45.iaa.csic.es:8080/ jmaiz/research/GOS/GOSmain.html
} 
Table 1

Stellar and Observational Data for Ofc and Of?p Stars

\begin{tabular}{|c|c|c|c|c|c|c|c|}
\hline Name & R.A. $(2000.0)$ & Decl. $(2000.0)$ & Spectral Type & V & $B-V$ & Observatory & Date \\
\hline \multicolumn{8}{|c|}{ Ofc } \\
\hline $\mathrm{CPD}-47=2963$ & 08.57 .54 .620 & $-47: 44: 15.71$ & O5 Ifc & 8.45 & +0.74 & $\mathrm{LCO}$ & 2008 May 22 \\
\hline Cyg OB2-11 & $20: 34: 08.514$ & $+41: 36: 59.42$ & O5.5 Ifc & 10.03 & +1.49 & САНА & 2008 Oct 15 \\
\hline Cyg OB2-9 & $20: 33: 10.734$ & $+41: 15: 08.25$ & O4.5 Ifc & 10.96 & +1.81 & САНА & 2008 Oct 14 \\
\hline Cyg OB2-8A & $20: 33: 15.078$ & $+41: 18: 50.51$ & O5 III $(\mathrm{fc})$ & 9.06 & +1.30 & CAHA & 2008 Jun 25 \\
\hline Cyg OB2-8C & $20: 33: 17.977$ & $+41: 18: 31.19$ & $\mathrm{O} 4.5 \mathrm{III}(\mathrm{fc})$ & 10.19 & +1.24 & CAHA & 2008 Jun 25 \\
\hline HD 93403 & $10: 45: 44.122$ & $-59: 24: 28.15$ & O5 III $(\mathrm{fc})$ & 7.27 & +0.21 & LCO & 2008 May 23 \\
\hline HD 93843 & $10: 48: 37.769$ & $-60: 13: 25.53$ & O5 III $(\mathrm{fc})$ & 7.31 & -0.03 & $\mathrm{LCO}$ & 2008 May 23 \\
\hline HD 93204 & $10: 44: 32.336$ & $-59: 44: 31.00$ & O5.5 V((fc)) & 8.44 & +0.09 & LCO & 2008 May 22 \\
\hline HDE 303308 & $10: 45: 05.919$ & $-59: 40: 05.93$ & O4 V((fc)) & 8.16 & +0.13 & $\mathrm{LCO}$ & 2008 May 23 \\
\hline HDE 319699 & $17: 19: 30.417$ & $-35: 42: 36.14$ & $05 \mathrm{~V}((\mathrm{fc}))$ & 9.62 & +0.79 & $\mathrm{LCO}$ & 2008 May 23 \\
\hline \multicolumn{8}{|c|}{ Of?p } \\
\hline NGC $1624-2$ & $04: 40: 37.266$ & $+50: 27: 40.96$ & O7f?p & 11.77 & +0.57 & САHA & 2009 Oct 30 \\
\hline HD 148937 & $16: 33: 52.387$ & $-48: 06: 40.47$ & O6f?p & 6.72 & +0.33 & $\mathrm{LCO}$ & 2008 May 21 \\
\hline \multirow[t]{2}{*}{ HD 191612} & $20: 09: 28.608$ & $+35: 44: 01.31$ & O6f?p & 7.77 & +0.26 & OSN & 2009 Jul 1 \\
\hline & & & O8fp & & & OSN & 2007 May 28 \\
\hline HD 108 & $00: 06: 03.386$ & $+63: 40: 46.75$ & O8fp & 7.38 & +0.16 & OSN & 2009 Sep 4 \\
\hline $\mathrm{CPD}-28=2561$ & $07: 55: 52.854$ & $-28: 37: 46.78$ & O6.5fp & 9.97 & +0.18 & $\mathrm{LCO}$ & 2008 May 23 \\
\hline
\end{tabular}

Notes. Most recent references to known Ofc binaries: Cyg OB2-9: van Loo et al. (2008), Nazê et al. (2008); Cyg OB2-8A: De Becker et al. (2004), component types O5.5 and O6; HD 93403: Rauw et al. (2002), component types O5.5 and O7.

Ofc association/cluster memberships in addition to Cyg OB2: Carina Nebula: HD 93204, HD 93403, HD 93843, HDE 303308; NGC 6334: HDE 319699 .

are listed in Table 1, while segments of the spectrograms are displayed in Figures 1 and 2 in the same order.

\section{RESULTS}

\subsection{The Ofc Stars}

A luminosity sequence of the new Ofc spectral category is shown in Figure 1. Although there were previous indications in some spectra (Walborn 1973b; Herrero et al. 1999; Walborn \& Howarth 2000), we were initially surprised to find strong C III $\lambda \lambda 4647-4650-4652$ emission lines in our data for four high-luminosity early-O stars belonging to the Cygnus OB2 association. However, as study of our large sample of high$\mathrm{S} / \mathrm{N}$ data proceeded, further examples of this phenomenon were encountered in other regions and at all luminosity classes, but always at or near spectral type O5, as seen in Figure 1. As shown by Walborn \& Fitzpatrick (1990, their Figures 4 and 5) and A. Sota et al. (2010, in preparation), although often present, the C III is much weaker than the N III at earlier and later spectral types. There are also some O4-O5 spectra in which, although still strong, the $\mathrm{C}$ III is weaker than the adjacent $\mathrm{N}$ III lines, e.g., in HD 15570, O4 If and HD 14947, O5 If. In some O4 spectra, the $\mathrm{C}$ III emission lines are joined by $\mathrm{C}$ IV $\lambda 4658$ emission of comparable intensity, e.g., HD 93250, O4 III(fc) and HD 15558, O4.5 III(fc); cf. Cyg OB2-9 in Figure 1 and see Walborn et al. (2002) for identification of the $\mathrm{C}$ IV line in $\mathrm{O} 2-\mathrm{O} 3$ spectra.

From investigation of the first $\sim 300$ spectra in our survey, 18 of types O3.5-O5.5 have been classified as Ofc so far. Of these, four are in Cygnus OB2, eight in the Carina Nebula complex, and two in IC 1805; while one in each are the only (optically bright) early-O stars in NGC 1893, NGC 6334, and NGC 6530. This subsample of our survey also contains 21 O4-O5 spectra not classified as Ofc; however, five of these are marginal cases with strong $\mathrm{C}$ III emission somewhat weaker than the $\mathrm{N}$ III, including two in the Carina Nebula and one in IC 1805. Others of these non-Ofe types are peculiar objects ( 5 Onfp, 2 O Iafpe). But two of them are the primary classification standards HD 46223,
O4 V((f)) and HD 46150, O5 V((f)) in NGC 2244, and another is the brightest early-O star in NGC 6611. These demographics suggest that a spectral type near O5 is a necessary but not sufficient condition for the Ofc phenomenon, which occurs primarily in certain associations and young clusters, but not others. Thus, a cluster parameter in addition to the stellar spectral type plays a role, such as initial rotational velocities (Maeder \& Meynet 2000), age, and/or chemical composition. These full samples and future ones will be presented and further discussed by A. Sota et al. (2010, in preparation) and in subsequent papers.

Some revisions and refinements of spectral categories and individual types will be noted in this discussion. Along with the recognition of the Ofc category, these are developments resulting from the quality and extent of our new survey data; they will be fully described and a comprehensive new spectral atlas will be presented in A. Sota et al. (2010, in preparation). For instance, the " + " parameter previously used to denote Si IV $\lambda \lambda 4089-4116$ emission in O-type spectra has been dropped, in view of the expanded array of selective emission lines that are now being recognized (Walborn 2001; Werner \& Rauch 2001; Walborn et al. 2004a; Corti et al. 2009). However, it has been deemed useful to add the " $\mathrm{c}$ " to emphasize $\mathrm{C}$ III emission in the new category discussed here, because of the prior definition of the Of phenomenon in terms of the adjacent $\mathrm{N}_{\text {III }}$ and $\mathrm{He}$ II $\lambda 4686$ emission lines only. The double and single parentheses introduced by Walborn (1971a) to describe weak N III emission in combination with strong $\mathrm{He}$ II absorption, and stronger N III with the He II weakened or neutralized, respectively, are retained in the Ofc types; the unadorned " $\mathrm{f}$ " or " $\mathrm{fc}$ " is reserved for all these features strongly in emission, and this progression is now recognized as a luminosity effect in normal spectra, with the last case corresponding to the supergiants.

\subsection{The Of?p Stars}

The Of?p notation was introduced by Walborn (1972) to describe two peculiar spectra (HD 108 and HD 148937) and 


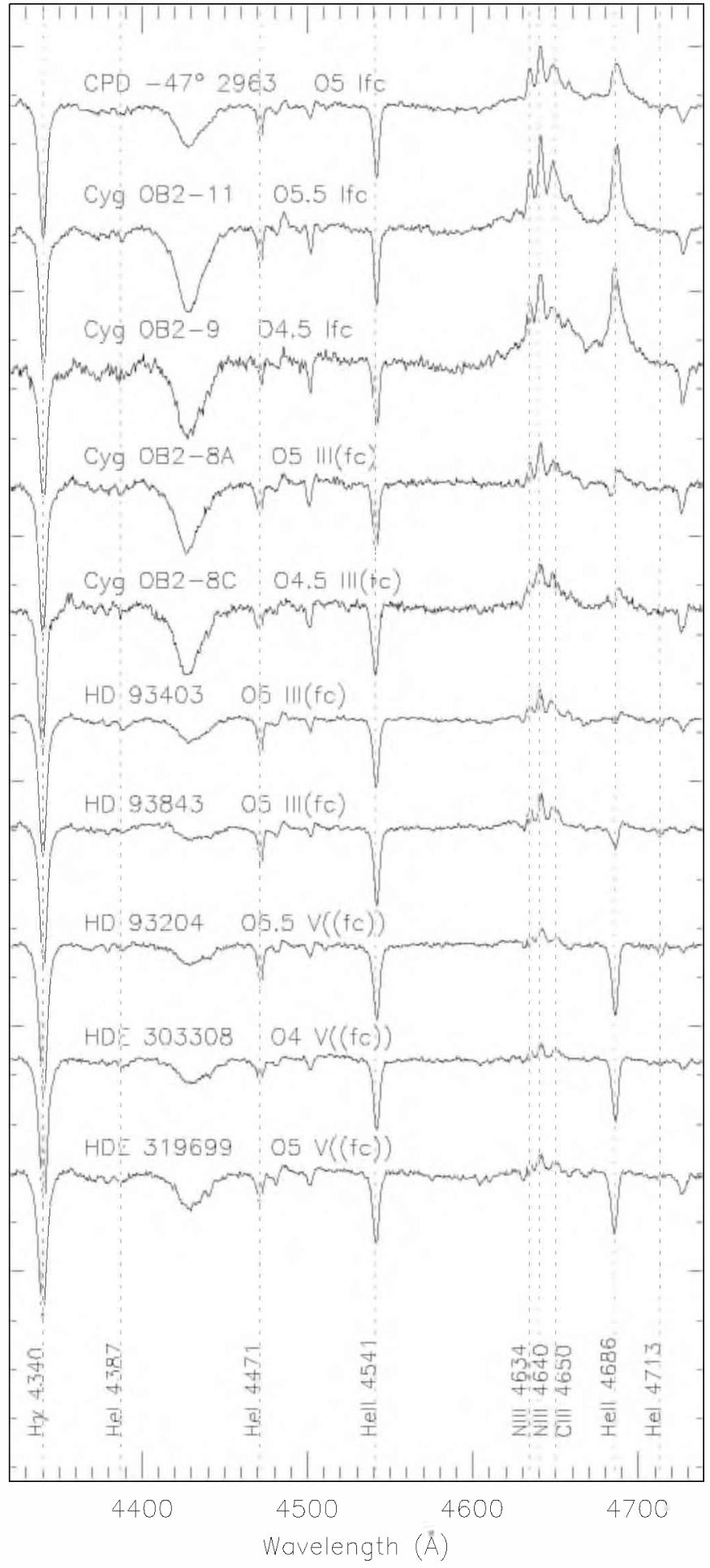

Figure 1. Luminosity sequence of Ofc spectra in the blue-green region. The ordinate is rectified intensity and the longer tick marks are separated by 0.5 continuum units. In addition to the stellar lines identified. the unidentified diffuse interstellar bands at $\lambda \lambda 4430,4502,4726$ are prominent.

emphasize doubt that they are normal supergiants, as the normal Of spectra had just been interpreted. A third example of the category (HD 191612) was discovered by Walborn (1973a). This distinction has been amply vindicated by subsequent developments. First, extreme, recurrent spectral variations were found in HD 108 (Nazé et al. 2001) and HD 191612 (Walborn et al. 2003); also, as shown in the latter paper, these stars do

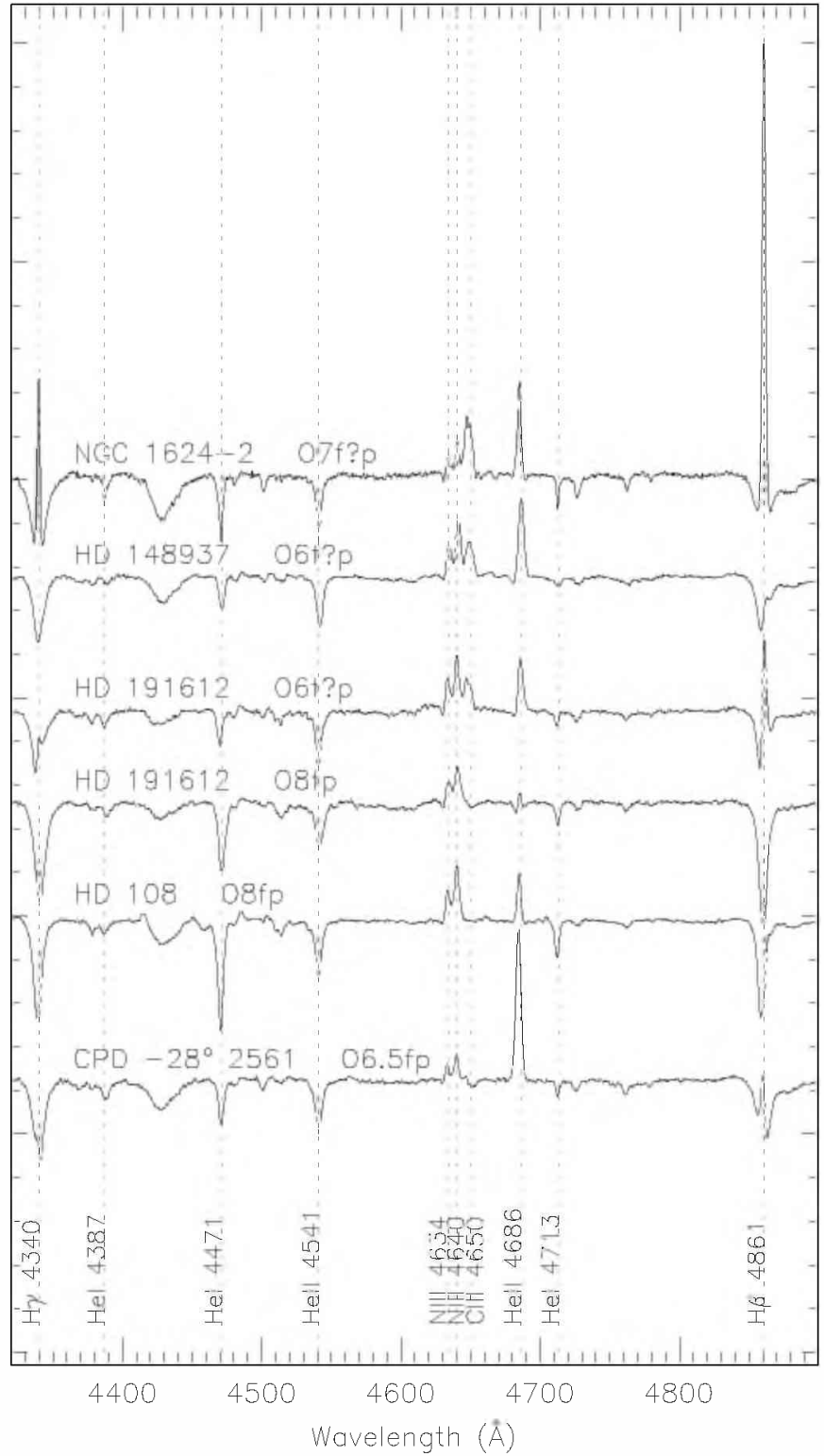

Figure 2. Spectra of the Galactic Of?p stars in the blue-green. See the Figure 1 caption for plot details.

not have supergiant wind profiles in the ultraviolet. A period in excess of 50 years is indicated for HD 108 (Nazé et al. 2001), whereas a strict period of 538 days was identified in HD 191612 (Walborn et al. 2004b; Howarth et al. 2007); these are now believed to be rotational periods. Then, HD 191612 (Donati et al. 2006) and HD 108 (F. Martins et al. 2010, in preparation) have become two of the first few O stars with detected magnetic fields, which most likely reveal their basic nature of braked, oblique rotators.

The most prominent defining characteristic of the Of?p category was the presence of C III $\lambda \lambda 4647-465()-4652$ emission lines of comparable intensity to the $\mathrm{N}$ III $\lambda \lambda 4634-464()-4642$. However, it is important to note that there were others, including sharp absorption, emission, and $\mathrm{P}$ Cygni features at $\mathrm{H}$ and He lines indicative of circumstellar structures, and a general lack of other Of supergiant properties such as prominent $\mathrm{Si}$ IV absorption lines and broad emission pedestals beneath the narrow emission lines. Now, as shown in the references cited and Figure 2, it has been found that the $\mathrm{C}$ III emission even disappears 
entirely at the late-type or "minimum" phases of HD 108 and HD 191612! It was fortuitous that both of these stars were near their maxima during the observations by Walborn (1973a).

Figure 2 shows a key segment in the spectra of the three previously known Galactic Of?p stars (with HD 108 appearing in its current minimum state and HD 191612 near both extremes of its variability range), together with two newly recognized similar objects that extend the phenomenology of the category.

The star NGC 1624-2 is the brightest in the ionizing cluster of the small, distant anticenter H II region Sh2-212 (Sharpless 1959; Moffat et al. 1979). ${ }^{9}$ We have found that it has an extreme, so far unique Of?p spectrum, with the C III emission blend substantially stronger than the N III features. The twodimensional spectral images show that the strong Balmer emission is definitely circumstellar and not from the $\mathrm{H}$ II region; a few repeated observations indicate that it moves with respect to the underlying absorption lines (or vice versa), with a small amplitude and a timescale of days, but further observations are ongoing to better characterize this behavior.

CPD $-28^{\circ} 2561$ was already classified as a peculiar Of object of undetermined nature by Walborn (1973a) and Garrison et al. (1977). Repeated high-resolution observations, associated with the GOSSS for the investigation of spectroscopic binaries (in the south, the Galactic O and WN [OWN] Survey, R.C.G. and R.H.B., Co-PIs), now show that this star undergoes extreme spectral transformations very similar to those of HD 191612, on a timescale of weeks (R. H. Barbá et al. 2010, in preparation). The C III $\lambda \lambda 4647-4650-4652$ emission intensity has not yet been seen to rival that of $\mathrm{N}$ III $\lambda \dot{i} 4634-464()-4642$, but it is definitely variable and the maximum may not yet have been covered.

\section{DISCUSSION}

Selective emission lines in O-type spectra, i.e., lines that come into emission while others from the same ions remain in absorption (Walborn 2001) are potentially a very powerful but as yet poorly developed diagnostic of physical conditions in the atmospheres of these stars, as well as of anomalous level populations in those ions. It is well established that these emission lines display smooth correlations with the stellar spectral types and luminosity classes, and that they have the same profiles as the stellar absorption lines in normal spectra, i.e., they arise in the stellar photospheres. However, the only one of the growing array of these features that has been subjected to detailed theoretical investigation in the literature is the $\mathrm{N}$ III $i \lambda 4634-464()-4642$ triplet, long ago by Mihalas et al. (1972) and Mihalas \& Hummer (1973). Corti et al. (2009) provided preliminary evidence that current state-of-the-art atmospheric models can reproduce these lines, or not, depending upon quite small changes in the basic parameters, but an effort to do so systematically and understand the atomic physics involved is as yet lacking.

The behavior of the C III $\lambda i 4647-465$ ()-4652 triplet in the Ofc spectra reported here constitutes a new selective emission effect, which contrasts sharply with the well-known C III $\lambda 5696$ singlet emission. While the former is very sharply peaked at

\footnotetext{
9 Currently this object can be identified in SIMBAD either as MFJ Sh 2-212 2 or as GSC 03350-00255. Although SIMBAD reports them as different objects separated by 1.4 , the two identifiers refer to the same star; SIMBAD further confuses the issue by calling the latter NGC 1624-1, as do Chini \& Wink (1984). See Deharveng et al. (2008) for a definitive identification chart, as well as an interesting study of the region. NGC 1624-2 is also 2MASS $04403728+5027410$.
}

spectral type O5, the latter pervades nearly the entire O-type domain (Conti 1974, Walborn 1980). The triplet absorption versus singlet emission dichotomy in these C III lines was already described by Swings \& Struve (1942) in the spectra of massive O stars, while Smith \& Aller (1969) and Heap (1977) discussed analogous effects in Of central stars of planetary nebulae. Physical explanations of these distinct behaviors will be of considerable interest. They are unlikely to be solely chemical abundance effects, because the spectral types are based on $\mathrm{He}$ ionization ratios, and the luminosity classes on the absolute strengths of the He II, N III, and as of now in some spectra near O5, C III emission lines. Nevertheless, there may be a secondary role of abundances, whether initial or from mixing of CNOcycled material in the stars themselves, to explain the systematic differences among associations and clusters discussed in the previous section. However, this behavior is quite different from that of ON versus OC spectra (Walborn 1976, 2003; Walborn \& Howarth 2000), which show opposite anomalies in $\mathrm{N}$ versus $\mathrm{C}$, O that correlate with the He abundance and certainly result from differing degrees of mixing or binary transfer of CNOprocessed material into the atmospheres and winds: absent in the $\mathrm{OC}$, present in normal spectra, and extreme in the ON (Smith \& Howarth 1994, Maeder \& Conti 1994). As always, the morphology provides strong hypotheses for subsequent physical investigation.

The Of?p stars are also N-rich (Nazé et al. 2008). In these peculiar objects, the phenomenology indicates that the C III $\lambda \lambda 4647-4650-4652$ emission is localized to some particular region of the unknown circumstellar structures, which is sharply occulted at certain rotational phases. However, the C III $\lambda 5696$ emission remains constant as a function of phase (Walborn et al. 2004b), and thus arises in a different location, most likely the stellar atmospheres. The strong dependence of the $\lambda \lambda 4647-465()-4652$ emission on spectral type (and thus, likely on the atmospheric parameters) in the Ofc stars may be related to its extreme spatial localization in the Of?p circumstellar environments. Thus, a physical explanation of one of these categories may illuminate that of the other.

N.R.W. thanks the Instituto de Astrofísica de Andalucía and its staff for kind hospitality and subsistence (through Spanish Government grant AYA2007-64052) during visits there in 2008 and 2009. His travel to Spain was supported by NASA through grants GO-10898.01 and GO-11212.03 from STScI. A.S., J.M.A, and E.J.A. acknowledge support by the Spanish Government Ministerio de Ciencia e Innovación grant AYA2007-64052 and by the Junta de Andalucía grant P08-TIC4075; J.M.A. is also supported by the Ramón y Cajal Fellowship program, cofinanced by the European Regional Development Fund (FEDER). R.H.B. acknowledges partial support from Universidad de La Serena Project DIULS CD08102. J.I.A. is supported by European Southern Observatory-Chilean Government Joint Committee funds. We are grateful for generous allocations of observing time at OSN, CAHA, and LCO, without which a program of this magnitude would not be feasible. Publication was supported by the STScI Director's Discretionary Research Fund. The Space Telescope Science Institute is operated by the Association of Universities for Research in Astronomy, Inc., under NASA contract NAS5-2655.

\section{REFERENCES}

Abt, H. A., Meinel, A. B., Morgan, W. W., \& Tapscott, J. W. 1968, An Atlas of Low-Dispersion Grating Stellar Spectra 
Chini. R.. \& Wink. J. E. 1984. A\&A. 139. L5

Conti. P. S. 1974. ApJ, 187, 539

Conti. P. S. \& Alschuler, W. R. 1971. ApJ, 170, 325

Conti, P. S., \& Leep, E. M. 1974, ApJ, 193, 113

Corti, M. A.. Walborn, N. R., \& Evans. C. J. 2009, PASP, 121, 9

De Becker. M.. Rauw. G.. \& Manfroid. J. 2004, A\&A, 424, L39

Deharveng, L., Lefloch, B., Kurtz. S., Nadeau, D., Pomarès, M., Caplan, J., \& Zavagno. A. 2008, A\&A, 482, 585

Donati, J.-F., Howarth, I. D., Bouret, J.-C., Petit, P., Catala, C.. \& Landstreet, J. 2006. MNRAS, 365, L6

Garrison. R. F.. Hiltner. W. A.. \& Schild. R. E. 1977. ApJS. 35. 111

Heap, S. R. 1977, ApJ, 215, 609

Herrero, A., Corral, L. J., Villamariz, M. R., \& Martín, E. L. 1999, A\&A, 348, 542

Howarth. I. D.. et al. 2007. MNRAS. 381. 433

Johnson. H. L., \& Morgan. W. W. 1953, ApJ, 117, 313

Maeder, A., \& Conti, P. S. 1994, ARA\&A. 32, 227

Maeder, A., \& Meynet, G. 2000, ARA\&A, 38, 143

Maíz Apellániz. J.. Walborn. N. R.. Galué. H. A.. \& Wei. L. H. 2004. ApJS. 151. 103

Mihalas, D., \& Hummer, D. G. 1973, ApJ, 179, 827

Mihalas, D., Hummer, D. G., \& Conti, P. S. 1972, ApJ, 175, L99

Moffat, A. F. J., FitzGerald. M. P., \& Jackson, P. D. 1979. A\&AS, 38, 197

Morgan. W. W.. \& Keenan. P. C. 1973, ARA\&A. 11. 29

Nazé, Y., De Becker, M.. Rauw. G.. \& Barbieri, C. 2008, A\&A, 483. 543

Nazé, Y., Vreux, J.-M., \& Rauw, G. 2001, A\&A, 372, 195

Nazé, Y., Walborn, N. R., \& Martins, F. 2008, RevMexAA. 44, 331

Plaskett. J. S.. \& Pearce. J. A. 1931. Publ. Dom. Astrophys. Obs.. 5. 99

Rauw. G.. Vreux. J.-M.. Stevens. I. R., Gosset. E.. Sana. H.. Jamar. C.. \& Mason. K. O. 2002, A\&A, 388, 552
Sharpless. S. 1959. ApJS. 4. 257

Smith. L. F. \& Aller. L. H. 1969. ApJ, 157, 1245

Smith. K. C. \& Howarth. I. D. 1994, A\& A. 290.868

Swings, P.. \& Struve, O. 1942, ApJ, 96, 254

van Loo, S., Blomme, R., Dougherty. S. M., \& Runacres, M. C. 2008, A\&A. 483,585

Walborn, N. R. 1971a, ApJS, 23, 257

Walborn, N. R. 1971b, ApJ, 167, L31

Walborn, N. R. 1972, AJ, 77, 312

Walborn, N. R. 1973a, AJ, 78, 1067

Walborn. N. R. 1973b. ApJ, 180. L35

Walborn, N. R. 1976, ApJ, 205, 419

Walborn, N. R. 1980, ApJS, 44, 535

Walborn. N. R. 2001, in ASP Conf. Ser. 242. Eta Carinae \& Other Mysterious Stars. ed. T. Gull. S. Johansson. \& K. Davidson (San Francisco. CA: ASP). 217

Walborn, N. R. 2003, in ASP Conf. Ser. 304, CNO in the Universe, ed. C. Charbonnel, D. Schaerer, \& G. Meynet (San Francisco, CA: ASP), 29

Walborn. N. R. 2009. in Stellar Spectral Classification. ed. R. O. Gray \& C. Corbally (Princeton. NJ: Princeton Univ. Press), 66

Walborn, N. R., \& Fitzpatrick. E. L. 1990, PASP, 102, 379

Walborn, N. R., \& Howarth, I. D. 2000, PASP. 112. 1446

Walborn, N. R., Howarth, I. D., Herrero, A.. \& Lennon, D. J. 2003, ApJ, 588. 1025

Walborn. N. R.. Morrell. N. I.. Howarth. I. D., Crowther. P. A.. Lennon. D. J.. Massey, P. \& Arias. J. I. 2004a, ApJ, 608, 1028

Walborn, N. R., et al. 2002, AJ, 123, 2754

Walborn, N. R., et al. 2004b, ApJ. 617, L61

Werner. K.. \& Rauch. T. 2001. in ASP Conf. Ser. 242. Eta Carinae \& Other Mysterious Stars, ed. T. Gull. S. Johansson, \& K. Davidson (San Francisco. CA: ASP), 229 